\title{
Decentralised Control and Peer-To-Peer Cooperation in Smart Energy Systems
}

\author{
Geert Deconinck
}

\begin{abstract}
In order to achieve a decarbonised energy system, change has to happen from electricity generation to the transmission grid over the distribution level all the way down to the industrial loads and the local households. To get involvement of communities in this energy transition, local participation is needed, so that the citizens can be aware of the impact of their energy-related actions on environment and climate. However, the energy system has typically been organised in a top-down fashion, with centralised approaches and little active control, resulting in passive grid and ditto customers. Smart grids have put active customers and consumer engagement as one of the cornerstones of a more intelligent energy infrastructure, which can be organised differently. Indeed, in different niches decentralised approaches have been used successfully (decoupled microgrids, peer-to-peer networks, etc.). This chapter explores how decentralised approaches can fit the future energy system and how it can empower people for engaging in the energy transition.
\end{abstract}

\section{The Rise of Local Energy Communities}

Decentralisation is becoming an important paradigm within energy systems. This happens not only at the physical level, where electricity generation decentralises by the widespread introduction of photovoltaic installations or other distributed energy resources or where heat is produced locally, close to where it is used. Also, data infrastructures become omnipresent with embedded monitoring and control systems, allowing for local smart energy applications (such as microgrids, virtual power plants or home energy management systems). Besides the technical aspects also policy opens up to local initiatives, in order to get the energy user more engaged in the energy transition and to get radical innovations (Lavrijssen \& Carrillo Parra, 2017).

This decentralisation paves to way towards local energy communities, and-in its most distributed version-to peer-to-peer energy trading. In a local energy community, a group of households or consumers shares some energy assets (like a battery

\footnotetext{
G. Deconinck ( $₫)$

KU Leuven/EnergyVille, Leuven, Belgium

e-mail: geert.deconinck@kuleuven.be

(C) The Author(s) 2021

M. P. C. Weijnen et al. (eds.), Shaping an Inclusive Energy Transition, https://doi.org/10.1007/978-3-030-74586-8_6
} 


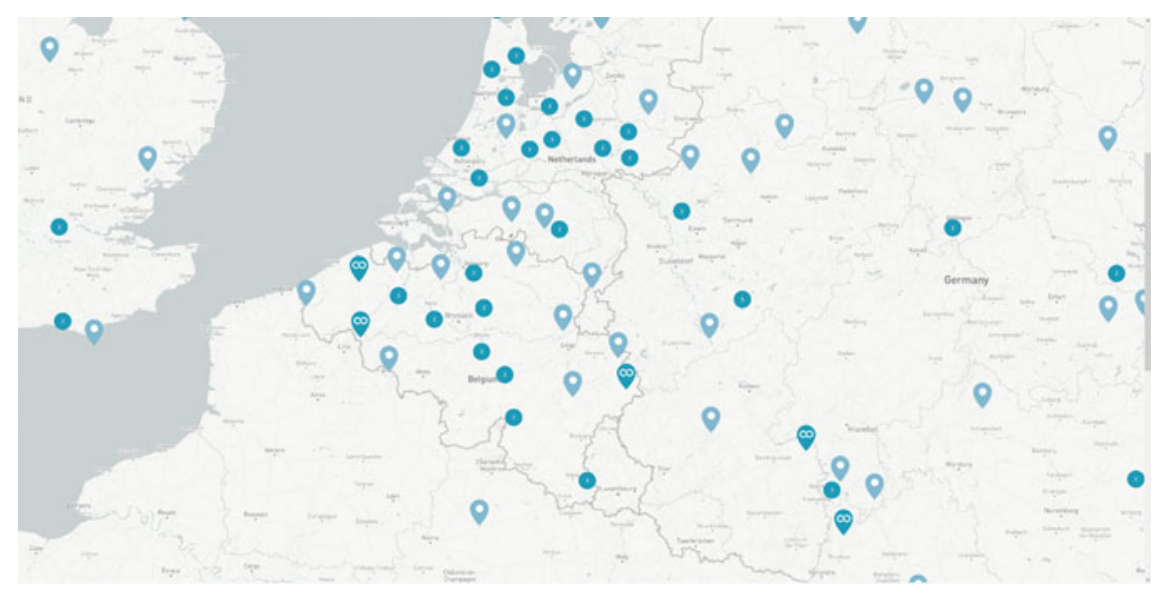

Fig. 1 Energy cooperatives in Belgium and in parts of the Netherlands, France, UK and Germany, that are member of REScoop (REScoop.eu (2020))

storage system or a heat buffer, or some electricity generation devices) and they organise themselves independently from supplier or distribution system operator. In a peer-to-peer energy system, customers buy and sell energy directly among each other. Such energy communities are often organised in energy cooperatives as legal entities.

Approximately 1,500 of these European energy cooperatives, representing about 1,000,000 citizens are gathered in the Renewable Energy Cooperatives (REScoop) (REScoop.eu, 2020) (Fig. 1). It REScoop describes energy communities as "a way to organise citizens that want to cooperate together in an energy-sector related activity based on open and democratic participation and governance, so that the activity can provide services or other benefits to the members or the local community. [...] The primary purpose of energy communities is to create social innovation - to engage in an economic activity with non-commercial aims." (REScoop.EU, 2019). As an example, some of the cooperatives invest in photovoltaic or wind generation for their participants, or organise joint buying of insulation material to improve energy efficiency, others cooperate with social housing companies to provide energy services or to renovate the building stock.

Although some forms of fully decentralised control (such as directly selling electricity to your neighbour over the public domain) are not (yet) allowed from a regulatory perspective in many countries, it is clearly the way forward. Such decentralisation has also been proposed in 2018 by the European Commission in its 'Clean Energy for all Europeans' package, which defines 'Citizen Energy Communities' as one of the cornerstones in the energy transition. ${ }^{1}$ Citizen Energy Communities are

\footnotetext{
${ }^{1}$ In that same package, also Renewable Energy Communities are defined in the recast Renewable Energy Directive (EU) 2018/2001, in article 2(16); see (REScoop.EU, 2019) for the subtle differences between these 'citizen energy communities' and 'renewable energy communities'.
} 
explicitly mentioned in article 2(11) of the recast Electricity Directive (EU) 2019/944 (European Parliament, 2019). They are defined as "a legal entity that:

- is based on voluntary and open participation and is effectively controlled by members or shareholders that are natural persons, local authorities, including municipalities, or small enterprises;

- has for its primary purpose to provide environmental, economic or social community benefits to its members or shareholders or to the local areas where it operates rather than to generate financial profits; and

- may engage in generation, including from renewable sources, distribution, supply, consumption, aggregation, energy storage, energy efficiency services or charging services for electric vehicles or provide other energy services to its members or shareholders." (European Parliament, 2019).

Much of the European policy needs to be translated into country-specific implementations, and not all member states are equally front-running in the energy transition. Roberts and Gauthier reviewed all National Energy and Climate Plans (NECP) of the European member states for their view on energy communities and they conclude, as an understatement, that there is much room for improvement (Roberts \& Gauthier, 2019). Their key takeaways are that awareness in EU member states on energy communities is moderate but actual planning is very low. At the positive end, a few member states (such as Greece) show strong commitment and their NECP has a comprehensive treatment of energy communities, including defined targets and detailed policies and measures. At the negative end, other member states (such as Estonia, Germany, Malta and Sweden) completely ignore energy communities in their NCEP. In between, many NECPs suffer from ambiguity around energy communities and they fail to distinguish them from distinct activities such as (individual) self-consumption. They conclude by stating that in the NECPs, "renewable energy communities and self-consumption overshadow other dimensions where energy communities can contribute: energy efficiency, energy poverty, ownership of distribution network, e-mobility, rural development, district heating, etc." (Roberts \& Gauthier, 2019).

Often the energy cooperatives or local energy communities are actively looking for a role in the traditional energy landscape, where distribution system operators as regulated entities often take up a rather conservative role. In such context, these innovative communities have to find their place between existing players (such as suppliers or retailers) and new players (energy service providers, aggregators, flexibility service providers, etc.).

All of these evolutions push also the control in the smart energy system towards lower and more decentralised levels. 


\section{Bottom-Up Control and Top-Down Control Meeting in the Middle}

Traditionally the power system has been controlled in a centralised way, with a prominent role for the system operator (e.g., the transmission and distribution system operator in a European context, or the independent system operator in a North-American context). Their role is to organise markets, and make sure that the operations are executed fluently by activating the necessary reserve power plants or decreasing power production to keep the grid balanced (ENTSOE, 2019). Keeping the frequency and voltage at their nominal levels and avoiding congestions are important assignments for this top-down control paradigm. While frequency is mainly a transmission grid problem, voltage and power quality issues appear everywhere, also at distribution level. However, at the distribution level (medium and low voltage level), there are not many controllable actuators, and the grid is often operated in quite a passive way. The distribution system operator has control over a number of devices in the substations in order to change configurations (connecting feeders and loads to particular bus bars), to change voltage levels (by changing the transformation ratio of transformers), or to change protection settings (to change selectivity in the protection). However, there is barely any control possible beyond the substations in the feeders. Even the monitoring at that level is limited as there are little or no sensors (fault indicators, voltage sensors, power quality measurements) in the feeders and sub-feeders. If a problem occurs, e.g., leading to a local blackout, the distribution system operator often has to wait until consumers call the service centre, and crew has to be sent in the field in order to solve the problems.

The trend towards smart grids implies that also the low and medium voltage grid gets equipped with sensors for monitoring and actuators for control. Because of this, faults can be detected earlier and some problems can be solved remotely without having to send crew in the field, so that the downtime for customers can decreased. Still this control is very much top-down and centralised in the operation room of the distribution system operator.

With more renewables at the customer's premises, also control has been added to that local level: the inverters that connect e.g., photovoltaics to the home, have a local embedded controller that continuously measures the grid voltage and disconnects the inverter in case there is an overvoltage. The national or regional grid codes specify (e.g., (Synergrid, 2017)) at which voltage level small photovoltaic systems (less than $10 \mathrm{kVA}$ ) need to disconnect. For larger installations, the ratio between active and reactive power supplied by the inverter can be used to support the voltage in the grid to a larger extent, and also here distribution system operators prescribe particular grid support requirements.

However, when active customers are envisioned, there is much more need (and opportunity) for monitoring and control at the local level, even behind the pointof-common-coupling, i.e., behind the meter that connects an individual customer to the grid. This is a fortiori the case when a smart meter is installed, which digitally measures off-take from the grid and injection in the grid, and has the possibility 
to communicate this information to a data operator, or to the distribution system operator, from where third parties (e.g., suppliers or retailers) have access to it. Smart meters allow for better monitoring or, if connected to actuators, for control applications as well. First, the smart meter allows to measure local power quality issues, or allows obtaining a local load profile from which the customer can benefit when negotiating a contract with his supplier. Additionally, local flexibility (at a residential level or at an industrial customer connected to the low or medium voltage grid) can be remotely controlled by an aggregator, which valorises it on the energy market or on the ancillary services market. Such flexibility results from the ability to shift the electricity use over time, and this increase or decrease in local off-take has a value at an aggregated level (Vandael et al., 2013). Another example is provided by a battery owner, who can use the local controller that manages the charging and discharging of the battery, to maximise its self-consumption, or to use the battery flexibility for an aggregator. In general, these demand response applications provide a great opportunity to better match the demand of electricity to its supply (Deconinck $\&$ Thoelen, 2019). A good survey of the state-of-art on demand response applications can be found in (Shoreh et al., 2016; Siano, 2014).

Hence, in a future smart grid, one sees many different control architectures appearing in parallel. Some of them are top-down, as for the distribution system operator controlling reactive power settings of renewable energy sources, or the data operator reading out smart meter registers, or the aggregator controlling flexibility. Others are local controllers that control the battery or interact with a home energy management system. These are examples of bottom-up control systems that generate a local equilibrium state that need to be taken as granted (or as un-controllable) by the top-down control applications.

It is foreseen that the amount of such control structures will only increase in the future (as e.g., new regulations will allow to have a contract with different suppliers simultaneously - one for injection in the grid and one for grid offtake (Atrias, 2019). Having all of these control architectures in parallel (especially if they do not share a common communication infrastructure) does not provide an optimal use of resources. Given the enormous ecological footprint of information and communication technology, and the need for digital sobriety (Ferreboeuf, 2019), it would be better if some of these infrastructures could be shared.

Such shared communication and control infrastructure rely on interoperability. Interoperability can be defined as the ability of two or more devices from the same vendor, or different vendors, to exchange information and to use that information for correct cooperation (International Electrotechnical Commission, 2019). Such interoperability needs to be effective at different levels: at the level of the communication media and protocols, at the level of the information model, at the functionality level and at the business level. The Smart Grid Reference Architecture Model (Fig. 2) provides an adequate way to reason about such interoperability for smart grid applications, in a layered approach (Smart Grid Coordination Group CEN-CENELEC-ETSI, 2012).

Instead of having all these parallel control architectures, it would be beneficial if entities (smart meters, home energy management systems, batteries ...) would be 


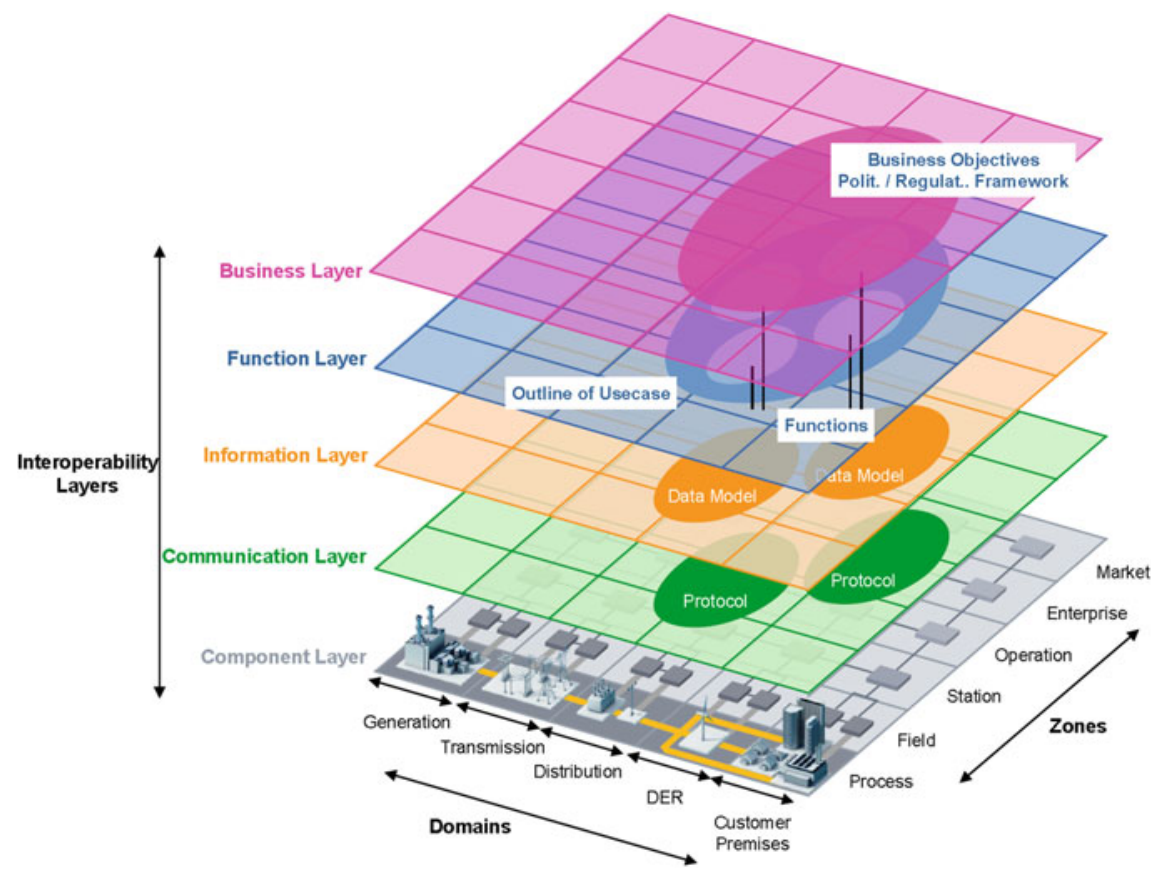

Fig. 2 SGAM framework (Smart Grid Coordination Group CEN-CENELEC-ETSI, 2012)

able to participate in different smart grid applications at the same time, because of an interoperable interface.

Additionally, from the viewpoint of the privacy of the end user, and of the confidentiality of its data, it would be better if local data is kept and processed locally, and is not transferred along the communication infrastructure several times for different applications. Local data provides better privacy protection than central data, and a single source for storing the data is less error prone than multiple sources for the same pieces data.

This interoperability would enable a more active engagement of the customer, including the ability to work with multiple market players at the same time (multiple suppliers, multiple aggregators, within a local energy community, etc.,). It hence enables end-customers to participate in multiple smart grid applications according to their needs, in an open market context.

This brings us to the question, which is the most appropriate distribution paradigm for the control actions, for which we introduce the taxonomy of Fig. 3. In a case with only local control, there is no communication between devices, and control decisions are taken only based on locally measured parameters (voltage, power, known price profiles, etc. Often it is beneficial however to coordinate control between entities, such that it is not only based on local sensor information, but also on information from other entities that is communicated to them. This can be done in different ways. 
Fig. 3 Relevant control paradigms for local and coordinated control (Deconinck \& Thoelen, 2019)

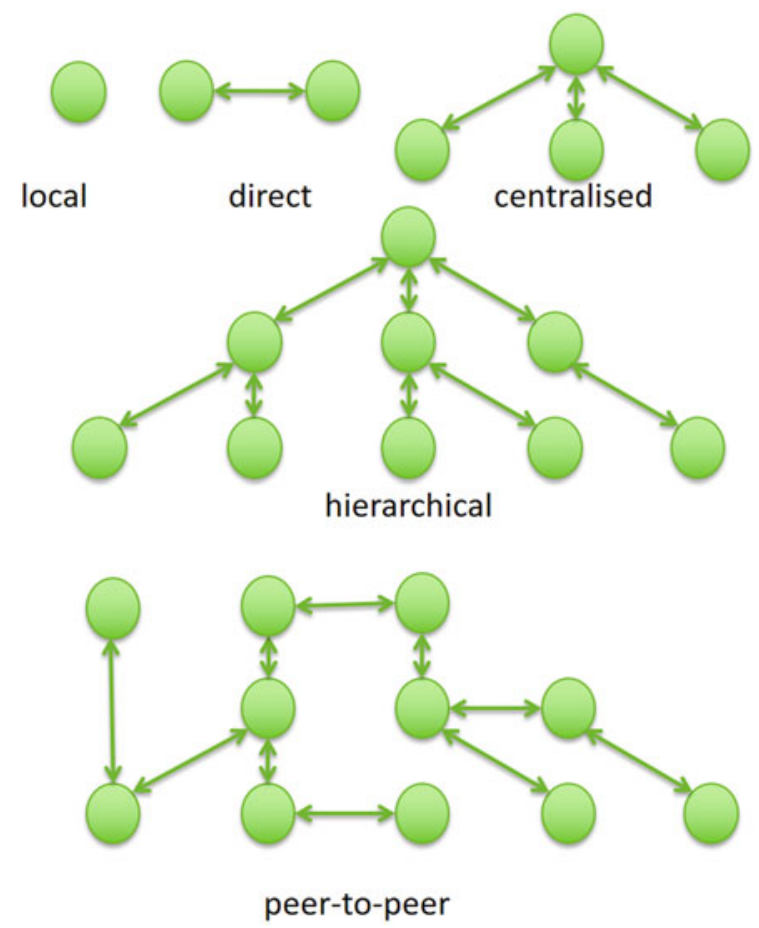

- In direct control there is a direct interaction between two entities, for instance an intelligent electric vehicle charger that determines the optimal moment to charge the car.

- With centralized control, a central agent controls all flexibility, for instance a distribution system operator that sends an interruption signal to specific loads to be shed.

- With a hierarchical approach, there are intermediate levels that ensure some scalability.

- Within a peer-to-peer approach, components only interact with some physical or logical neighbours in a flat hierarchy.

Table 1 indicates relevant differences and advantages of the different approaches.

\section{Going Completely Decentral: Peer-To-Peer}

Opposite to the centralised control, completely decentralised systems pop up at the other end of the spectrum: there, each unit is autonomous and collaborates with its neighbours, in a peer-to-peer fashion. More than a decade ago, it has been shown - both in simulations and in a practical implementation - that such completely 
Table 1 Advantages of centralised and decentralised control

\begin{tabular}{l|l}
\hline Centralised control & Decentralised control \\
\hline More simple & More scalable \\
\hline Single point of control & No single point of failure \\
\hline $\begin{array}{l}\text { SCADA-compatible } \\
\text { Requires dedicated communication } \\
\text { architectures }\end{array}$ & $\begin{array}{l}\text { Internet-compatible } \\
\text { Fits with many communication architectures: } \\
\text { overlay networks, peer-to-peer ... }\end{array}$ \\
\hline Onester/slave protocols & Publish/subscribe protocols \\
\hline More compatible with integrated energy & Each entity interfaces to multiple applications \\
companies with few actors & $\begin{array}{l}\text { More compatible to a liberalized, open market } \\
\text { model with many actors }\end{array}$ \\
\hline Passive customers & Engaged, active customers \\
\hline
\end{tabular}

distributed approaches are a feasible alternative to control a microgrid with distributed energy resources as an autonomous electricity network ( $A E N)$ without any central master (Vanthournout et al., 2005). In that microgrid, control was implemented as a combination of local droop control at the inverters, with a decentralised secondary control (to keep the voltage within its limits), and a decentralised tertiary control application (that prioritises the more economic energy resources) (Brabandere et al., 2007). The communication infrastructure was based on a peer-to-peer gossiping protocol that ran on top of a dynamic semantic overlay network (Deconinck \& Vanthournout, 2009).

Many more examples of decentralised or peer-to-peer control in smart grid applications do exist meanwhile. In (Almasalma et al., 2017), a distributed voltage control and optimisation method is proposed that optimises the reactive power settings of selected resources in a microgrid to keep the voltage quality within predefined limits. Also here, communication is fit for a decentralised approach, with a gossiping protocol to spread information between the active component, and with a distributed optimisation scheme based on dual decomposition to calculate the voltage set points. A hardware-in-the-loop implementation of this approach validated its performance, using a wireless device-to-device communication system (Almasalma, 2018). Its setup is represented in Figs. 4 and 5. This implementation showed that fully distributed voltage control systems can indeed provide satisfactory regulation of the voltage profiles.

A larger case study on a 62-bus and 124-bus network provided very acceptable results in terms of both convergence speed and optimality (Almasalma et al., 2019). 


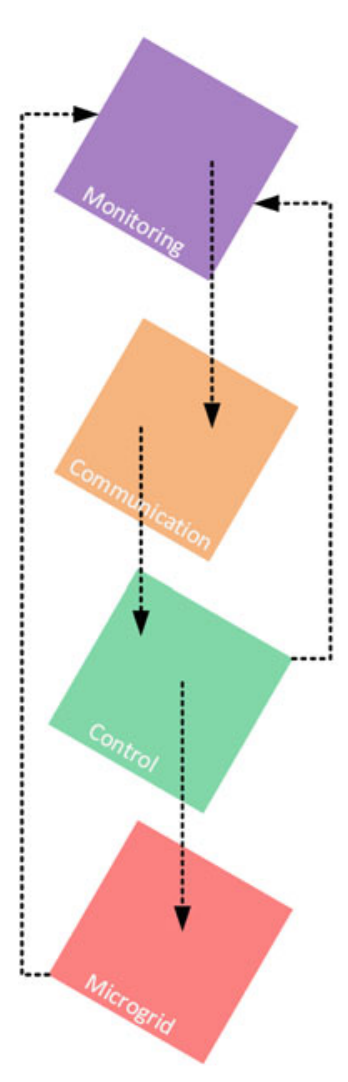

Testbed layers

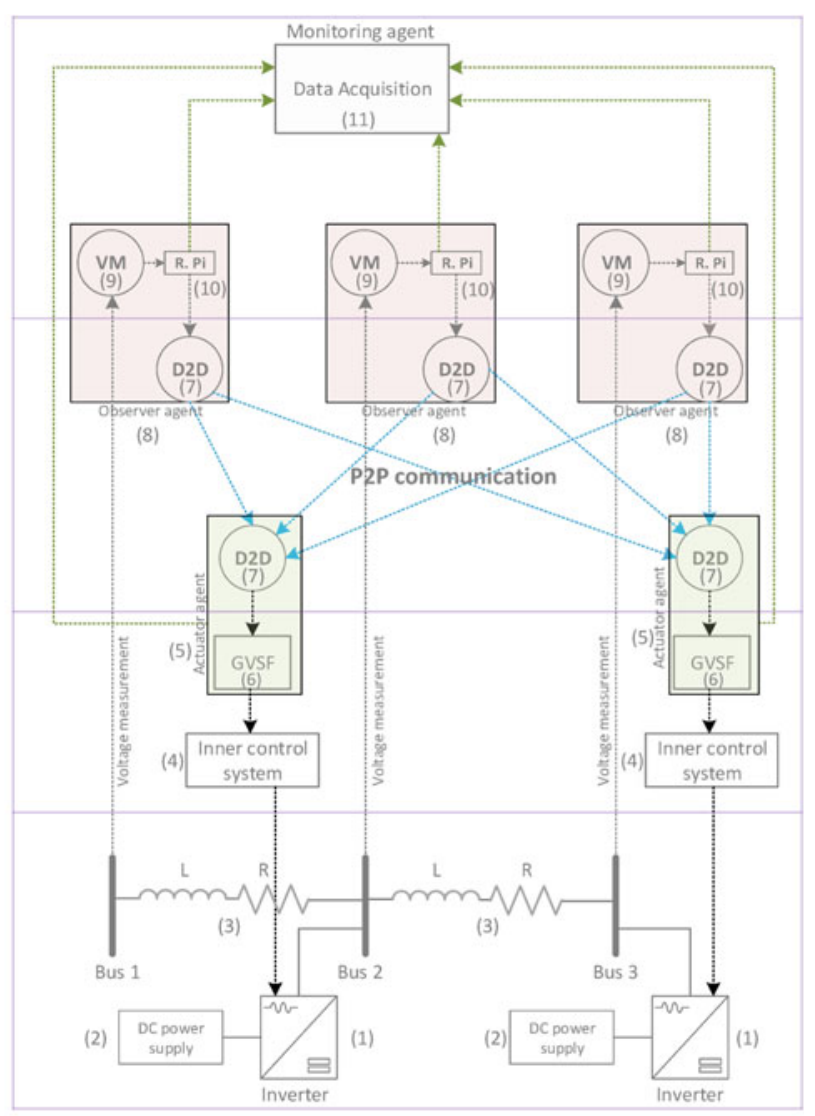

Interaction between the layers

Fig. 4 Multi-layer multi-agent architecture of the peer-to-peer (P2P) voltage control testbed (VM stands for voltmeter, D2D: device-to-device communication module, GVSF: grid voltage support function, R.Pi: raspberry pi computer, R: resistor, L: inductor, labels 1 to 11 indicate the different parts of the testbed) (Almasalma, 2018)

\section{Peer-To-Peer Control, Communication and Trading}

Not only the control architecture and communication architecture can be decentralised, also business applications can be run in a similar way. In the European H2020 project P2P-SmarTest (“P2P-SmarTest Project", 2019), energy trading between microgrids was seen as major example to develop a decentralised approach on the three levels: control, communication and trading (Pouttu et al., 2017). This energy trading is presented in Fig. 6. Also other researchers have investigated similar approaches of control and energy trading between autonomous entities, whether they call them microgrids, cells (multi-microgrids) or multi-cells (Zhang et al., , 2017, 2018). All of these levels provide different degrees of aggregation. 


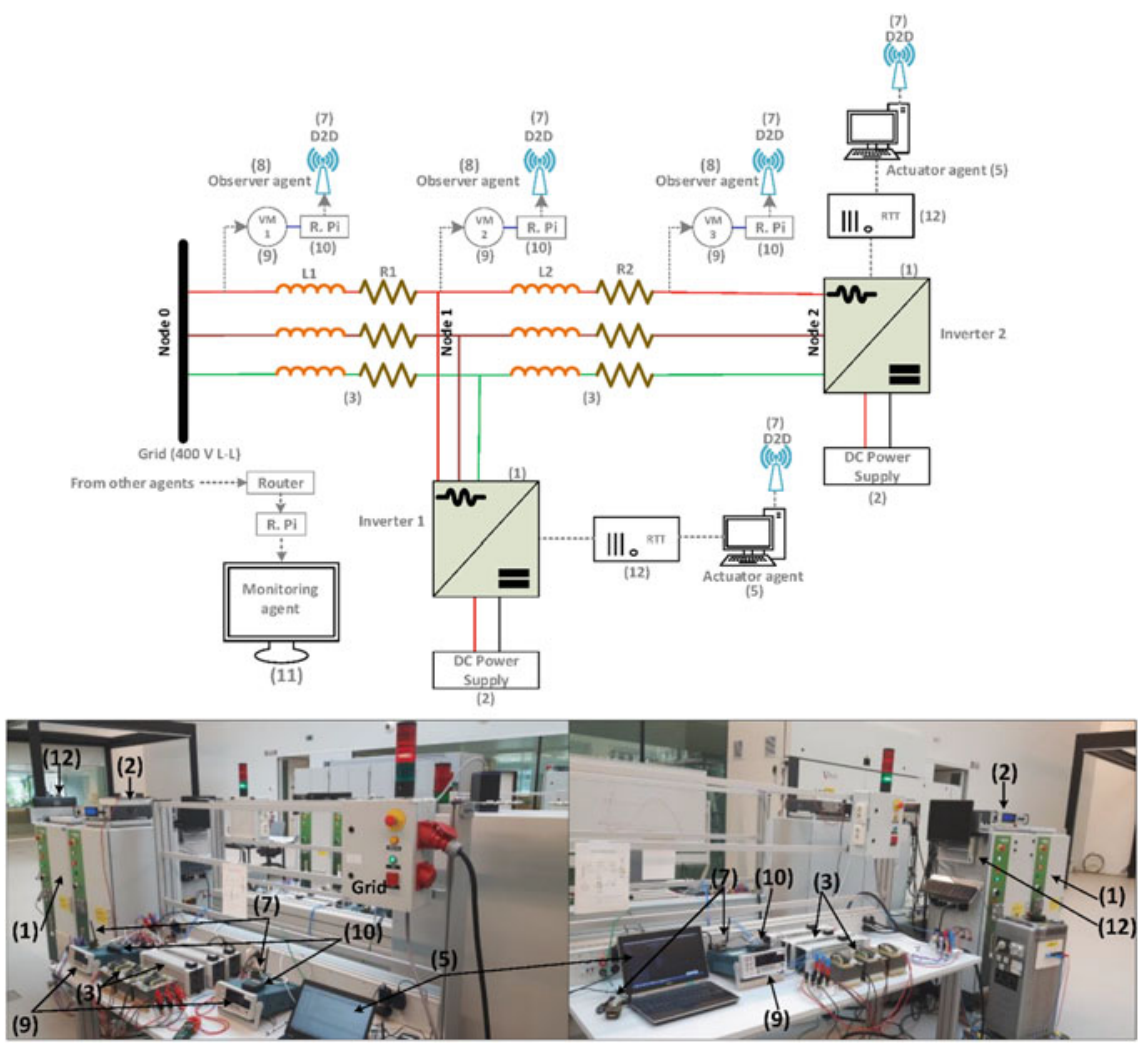

Fig. 5 Schematic of the peer-to-peer voltage control testbed (VM stands for voltmeter, D2D: device-to-device communication module, R: resistor, L: inductor, RTT: real time target computer, R.Pi: raspberry pi computer, labels (1)-(12) indicate the different parts of the testbed, labels (1)-(11) are the same as in Fig. 4) (Almasalma, 2018)

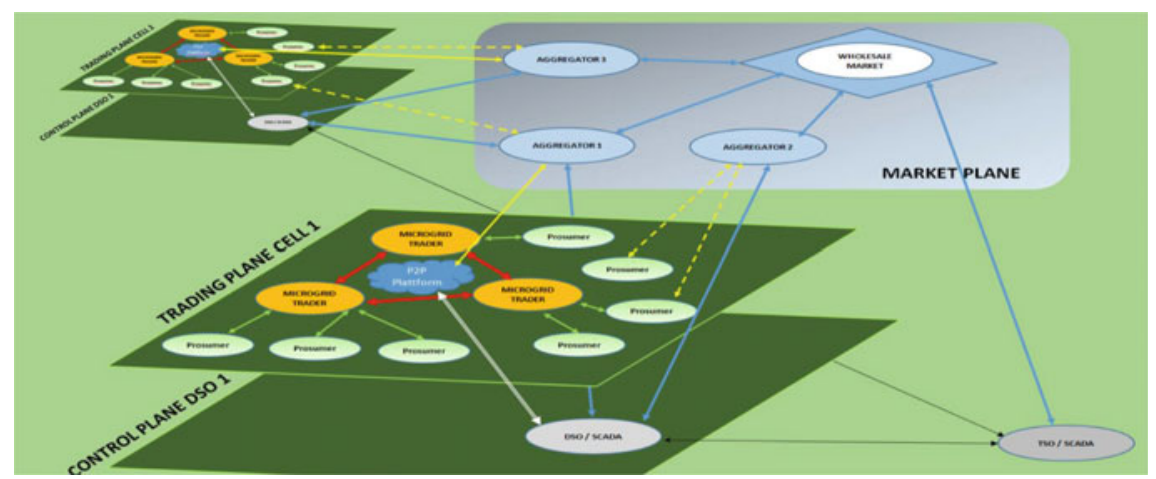

Fig. 6 Peer-to-peer energy trading in the P2P-Smartest project (Pouttu et al., 2017) 
Decentralised approaches also allow for different trust models and different ways to validate actions and transactions. In this context, distributed ledger technology that provides a consensus algorithm on top of peer-to-peer networks is an interesting alternative to centralised databases (Deconinck \& Vankrunkelsven, 2020). Well-known examples of this technology include blockchains (such as Ethereum), block directed acyclic graphs (such as RChain) and transaction-based directed acyclic graphs (such as Byteball), on top of which smart contracts can be implemented. Such smart contracts allow the performance of credible transactions without third parties, in a way that is irreversible, irrefutable and trackable. In the energy sector, different applications of blockchain and smart contracts are being developed (Mihaylov et al., 2018; Peter et al., 2019). In this context, the Users TCP (Technology Collaboration Programme) of the International Energy Agency (IEA) has set up a "Global Observatory on Peer-to-Peer, Community Self-Consumption and Transactive Energy Models" as "a forum for international collaboration to understand the policy, regulatory, social and technological conditions necessary to support the wider deployment of peer-to-peer, community self-consumption and transactive energy models" (GOP2P, 2021). It collects best practices and lessons learned of such decentralised models, beyond the technical perspective, from all over the world.

Having all these trends towards decentralisation, an interesting rhetoric question pops up, whether in a future smart grid, where the local resources in the microgrid are providing grid or where a microgrid manager takes over this responsibility of a central controller, it still makes sense to have a distribution system operator to run the grid? Alternatively, the grid codes and the active control units in the different devices are able to provide the same services. In our opinion, this thought experiment needs to be elaborated, in order to see the real minimal control requirements to be allocated to a central unit in a context of interoperable devices and peer-to-peer control applications. Especially if the distribution system operators are not eager to adapt to the new smart grid ecosystems, their role will be taken over by other players; Europe's Joint Research Centre provides a very good overview of the future-readiness of distribution system operators via its DSO Observatory (Prettico et al., 2019).

\section{Data-Driven Approaches and Machine Learning}

Together with the increase in data and data streams in a smart grid, we see a gradual shift in the control methodologies that are used in smart grid applications. Modelbased control and optimisation techniques have been used for decades, with very good results, also in an uncertain environment. Especially model predictive control, where a receding horizon is considered to base instantaneous control decisions upon, is a very powerful method. However, such model-based approach requires a model of the physical systems that are controlled. White box and grey box modelling use first principle methods or fit parameters to a reduced order representation of smart grid entities, and control and optimisation is often performed by a single controller. Data-driven approaches, on the contrary, do not require a physical understanding of 
the behaviour to build a model; rather the model can be learned from analysing the data, with techniques from supervised or unsupervised learning. Many applications of machine learning in demand response applications exist; a good overview is given in (Vázquez-Canteli \& Nagy, 2019). The main advantage is that the machine learning algorithms can easily fine-tune the models to the specifics of individual devices or can take uncertainty from user behaviour into account.

One step beyond, with data-driven approaches it is even not necessary any more to create a model first and then controlling it, but techniques such as reinforcement learning methods can learn a control policy directly. By adding a cost function (such as total energy use, greenhouse gas emissions or monetary cost), an optimal control policy can be found. In such a reinforcement learning method, exploration of the state space, and exploitation of the learned knowledge allow gradually improving the performance. Such reinforcement learning techniques have been successfully applied to different smart grid applications, such as controlling an electric boiler (Ruelens et al., 2018), a battery (Mbuwir et al., 2017), or a fleet of electric vehicles (Vandael et al., 2015).

However, all these data-driven applications and remote control abilities provide a number of threats as well and there is a clear need to ensure security of the application and privacy of the users.

- From a security perspective, the crucial properties are confidentiality, integrity and availability. Confidentiality can be ensured with the appropriate access control mechanisms and authentication procedures, together with encryption techniques. For integrity, adequate signature schemes and data checksum techniques can be used. Availability implies a robust information and communication technology infrastructure that performs adequately under varying load conditions. From a system's perspective, a defence-in-depth approach shall be followed, with multiple protection layers to detect and withstand intrusions, attacks or component failures.

- From a privacy perspective, when personal preferences or behaviour can be derived from the data, this can only be done when the user has given prior consent and when the European GDPR (General Data Protection Regulation) guidelines have been followed. However, a privacy-by-design approach is better and more robust than just GDPR-compliance. Such approach is based on different principles to deal with the data: minimise, hide, separate, aggregate, inform, control, enforce and demonstrate (Hoepman, 2014). As an example, it is not necessary to gather 15-min load profiles to only provide monthly billing, or it is not relevant what your gender or age is, in order to make a contract with an electricity supplier, as long as you are an adult that is legally allowed to sign a contract.

Evidently, some data (e.g., related to outages and power quality) are necessary to ensure the security of supply and to operate the grid smoothly, while other data are needed for commercial processes (e.g., consumed electricity per time-of-use period for billing).

According to the CEER (Council of European Energy Regulators), a lot needs still to be done to have the cybersecurity and privacy protection in the smart energy grids at the correct level (CEER, 2018). Especially if data are collected at finer 
granularity, larger resolution and more often, privacy gets at stake. It is e.g., possible to derive customer habits (like building occupancy or appliance use) from fifteen-minute electricity consumption data, or to derive more detailed info such as appliance settings from sub-second resolution apparent power measurements (Labeeuw \& Deconinck, 2011).

For instance, data-driven approaches and machine learning techniques are often deployed to characterise the power flexibility from residential customers for demand response applications as they are able to consider multiple types of uncertainty, and such techniques lead to a better local flexibility characterisation and usage forecasting than approaches based on physical models (Vázquez-Canteli \& Nagy, 2019). However, in order to engage fully the residential end-user in this story it is of the utmost importance to guarantee their privacy, and compliance with Europe's GDPR regulation is only a first step into this. It is our firm conviction that keeping all data at the customer's side, and hence not transferring it nor centralising it e.g., at an aggregator, is a clear advantage and will decrease customer's suspicion and allow more end user engagement.

If the data is kept locally and not transferred to multiple centralised actors, the individual has much more control about the use of the data. Of course, it assumes that some data processing is done locally, when a central actor requires information. An example of such local data processing is provided by the calculation of the total injection and grid off-take over a billing period, which needs to be calculated by the smart meter instead of forwarding the full load profiles at a 15-min resolution to a data operator. Another example is the local calculation of the available flexibility from residential appliances for demand response to be used by an aggregator, rather than sending the raw data to this aggregator.

Also, in different other sectors (such as sharing data on multiple social networks), there have been proposals to keep data local, and not to provide all data to the central databases of the social networks providers. OpenPDS (Personal Data Storage) is a clear example of this (Montjoye et al., 2014): it keeps all personal data in a local storage, and provides only 'SafeAnswers' to the allowed third parties requesting access. From a privacy perspective, this gives much more control to the user, who can select which data to share with whom, or to easily delete particular data which is no longer relevant.

In a similar way, for smart grid applications, it might be useful to look into techniques related to computing on encrypted data, which has been studied well in the domain of cryptography. Indeed, many applications require data to remain private (healthcare data, financial data, etc.), although one or more external parties would need to compute a specific function on this data for research, socio-economic or commercial purposes. (Here, calculates the aggregated flexibility from a cluster of residential customers without revealing flexibility data from individuals.) Multiple approaches for computing on encrypted data have been proposed in the literature. Most relevant in the context of smart grid applications are homomorphic encryption and secure multi-party computation. 
- Although homomorphic encryption was conceptualized by Rivest, Adleman and Dertouzos more than 40 years ago already (Rivest et al., 1978), the construction of such a scheme that can compute 'complex' operations on encrypted data was an open problem until Gentry presented the first fully homomorphic encryption scheme using ideal lattices in 2009 (Gentry, 2009). However, this scheme and its variants were extremely slow and did not provide a practical solution. Therefore, research has focused on a more practical variant: somewhat homomorphic encryption, which is significantly more efficient in practice and can be used when the algorithm applied to the encrypted data is known in advance. Their main limitation is that they only allow performing a limited number of computational steps on the encrypted data.

- Multi-party computation allows multiple mutually distrustful parties to compute any function on their private inputs without disclosing those inputs to each other. Security in multi-party computation comes at a cost of efficiency. Every computation requires information exchange among the parties, which can become expensive for non-linear operations with a large multiplicative depth. Additionally, it depends on a non-collusion assumption: at least one party has to remain honest. Furthermore, the larger number of parties involved, the slower the protocol is, as more data needs to be transmitted among the parties. In recent years, practical implementation of general-purpose multi-party computation protocols have appeared and have been successfully applied in well-defined use cases, such as electricity load forecasting.

In the SNIPPET project (Montakhabi et al., 2020; SNIPPET—Secure \& Privacyfriendly Peer-to-peer Electricity Trading”, 2019), a secure and privacy-friendly peerto-peer energy trading system is being developed, based on such techniques for computing on embedded data.

Besides security and privacy in a smart grid context, one also needs to consider customer protection. For instance, several types of smart meters allow for a remote connection and disconnection of customers. However, it is not acceptable that a supplier would disconnect a non-paying customer and leave him without electricity in the cold and dark during winter. In Belgium, e.g., every customer must still be supplied with at least $6 \mathrm{~A}$ (yielding approximately $1.4 \mathrm{~kW}$ at $230 \mathrm{~V}$ ) by the distribution system operator, even if all suppliers dropped him because of not paying the bills. Such regulatory framework is needed in order to protect the rights of the customers (Deconinck et al., 2011). Other customers might by apathetic or not interested in the data-driven approaches; also these customers have the right for a good power quality and a balance has to be found between sharing the minimal data for a minimal functionality with more engaged approaches that provide more features. A good overview of the context of how European policy makers deal with energy poverty is found in (Bouzarovski, 2017). Other examples of customer protection rights include the requirement to be able to check in its home at the smart meter the registers that were used for billing - they have priority over the transmitted values to the premises of the data operator. 
However, the legal frameworks, at regional, national or supranational level for dealing with this customer protection, are not always up to date with the latest evolutions in smart grids technology.

\section{Conclusion: Power to the People}

In order to achieve a decarbonised energy system, changes are needed at all levels. At the transmission grids, one needs to reach out towards global grids, with power outlets on the sea for non-country specific generation; the distribution grid level needs to become an active and smart grid, rather than a passive infrastructure; the final customers will be organised in local households or in energy communities together with neighbours; independent industrial customers will make a microgrid with neighbouring companies, etc. This implies decentralised control besides the classical centralised control paradigms, combining the benefits of both approaches.

Energy communities can play a key role in decentralised control approaches. To get involvement of such communities in this energy transition, local participation is needed, so that the citizens and end users can be aware of the impact of their energyrelated actions on the environment and on climate. They need to have a relationship with the energy assets and have a feeling of impact. However, the energy system has typically been organised in a top-down fashion, with centralised approaches and little active control, resulting in passive, unaware and uninterested customers. Smart grids have the potential to put active customers and consumer engagement as one of the cornerstones of a more intelligent energy infrastructure, which can be organised differently, in a more distributed way.

It is our firm conviction that the energy infrastructure needs to be prepared for a paradigm shift from centralised top-down control to distributed bottom-up control and completely decentralised peer-to peer approaches. Indeed, in different niches decentralised approaches have been used successfully (decoupled microgrids, peerto-peer networks, etc.). The different examples in this chapter have explored how decentralised approaches can fit the future energy system, what are the related opportunities and threats, and how it can finally empower people for better engaging in the energy transition.

Acknowledgements This work has been partially supported by KU Leuven project C24/16/018, FWO project S007619N (SNIPPET), and VLAIO Flux50 projects HBC.2018.0527 (ROLECS) and HBC.2019.0073 (PrivateFlex).

\section{References}

Almasalma, H., et al. (2018). Experimental validation of peer-to-peer distributed voltage control system. Energies, 11(5), 1304-1325. https://doi.org/10.3390/en11051304 
Almasalma, H., Engels, J., \& Deconinck, G. (2017). Dual-decomposition-based peer-to-peer voltage control for distribution networks. CIRED—Open Access Processing Journal, 2017(1), 17181721. https://doi.org/10.1049/oap-cired.2017.0282

Almasalma, H., Claeys, S., \& Deconinck, G. (2019). Peer-to-peer-based integrated grid voltage support function for smart photovoltaic inverters. Applied Energy, 239, 1037-1048. https://doi. org/10.1016/J.APENERGY.2019.01.249

Atrias. (2019). "Atrias Market Model UMIG 6.5.1.16". https://www.atrias.be/UK/Pages/Publicati ons_UMIG65.aspx. Accessed July 11, 2019.

Bouzarovski, S. (2017). Energy poverty: (Dis) assembling Europe's infrastructural divide. Palgrave Macmillan.

CEER. (2018). "Cybersecurity report on Europe's electricity and gas sectors (C18-CS-44-04)," [Online]. Available: https://www.ceer.eu/documents/104400/-/-/684d4504-b53e-aa46-c7ca-949 a3d296124

De Brabandere, K., Vanthournout, K., Driesen, J., Deconinck, G., \& Belmans, R. (2007, June). "Control of microgrids". In 2007 IEEE power engineering society general meeting, (pp. 1-7). https://doi.org/10.1109/PES.2007.386042

de Montjoye, Y.-A., Shmueli, E., Wang, S. S., \& Pentland, A. S. (2014). OpenPDS: Protecting the privacy of metadata through safeanswers. PLoS ONE, 9(7), e98790. https://doi.org/10.1371/jou rnal.pone.0098790

Deconinck, G., \& Thoelen, K. (2019, August). "Lessons from 10 years of demand response research: Smart energy for customers?.” IEEE System Man, Cybernetic Magazine, 5(3):21-30. https://doi. org/10.1109/MSMC.2019.2920160

Deconinck, G., \& Vankrunkelsven, F. (2020, November). "Digitalised, decentralised power infrastructures challenge blockchains." Proceedings Institution of Civil Engineers-Smart Infrastructure and Construction, 1-12. https://doi.org/10.1680/jsmic.20.00013

Deconinck, G., \& Vanthournout, K. (2009). Agora: A semantic overlay network. International Journal Critical Infrastructures, 5(1/2), 175. https://doi.org/10.1504/IJCIS.2009.022855

Deconinck, G., Delvaux, B., De Craemer, K., Qiu, Z., \& Belmans, R. (2011). "Smart meters from the angles of consumer protection and public service obligations." In 16th International conference on intelligent system applications to power systems (ISAP). https://doi.org/10.1109/ISAP.2011. 6082207

ENTSOE. "Electricity balancing." https://www.entsoe.eu/network_codes/eb/. Accessed July 11, 2019.

European Parliament. (2019). 2029/944/EU Directive of the European parliament and of the council on common rules for the internal market for electricity and amending directive 2012/27/EU (recast).

Ferreboeuf, H. (2019). "Lean ICT—towards digital sobriety (The Shift Project)". [Online]. Available: https://theshiftproject.org

Gentry, C. (2009). "Fully homomorphic encryption using ideal lattices." Proceedings 41st ACM Symposium on Theory of Computing (STOC 2009), 9(2009):169-178.

GOP2P (2021). Global observatory on peer-to-peer, community self-consumption and transactive energy models (IEA - UsersTCP). https://userstcp.org/annex/peer-to-peer-energy-trading/. Accessed April 20, 2021.

Hoepman, J.-H. (2014). Privacy design strategies. (pp. 446-459). Springer.

International Electrotechnical Commission. (2019). "IEC 61850:2019 SER Communication networks and systems for power utility automation". https://webstore.iec.ch/publication/6028. Accessed July 11, 2019.

Labeeuw, W., \& Deconinck, G. (2011). "Non-intrusive detection of high power appliances in metered data and privacy issues." In Proceedings 25th conference on passive and low energy architecture of the energy efficiency in domestic appliances and lighting (EEDAL) conference, (pp. 1-7). 
Lavrijssen, S., \& Carrillo Parra, A. (July 2017). "Radical prosumer innovations in the electricity sector and the impact on prosumer regulation.” Sustainability, 9(7), 1207. https://doi.org/10.3390/ su9071207

Mbuwir, B. V., Ruelens, F., Spiessens, F., \& Deconinck, G. (2017). "Battery energy management in a microgrid using batch reinforcement learning." Energies, 10(11). https://doi.org/10.3390/en1 0111846

Mihaylov, M., Razo-Zapata, I., \& Nowé, A. (2018). “NRGcoin-A blockchain-based reward mechanism for both production and consumption of renewable energy." In Transforming climate finance and green investment with blockchains, (pp. 111-131). Academic Press.

Montakhabi, M. et al. (2020). "New roles in peer-to-peer electricity markets: Value network analysis." In 6th IEEE international energy conference (EnergyCon-2020), (p. 6).

P2P-SmarTest Project. https://www.p2psmartest-h2020.eu/. Accessed July 11, 2019.

Peter, V., Paredes, J., Rosado Rivial, M., Soto Sepúlveda, E., \& Hermosilla Astorga, D. A. (2019). Blockchain meets energy — digital solutions for a decentralised and decarbonized sector. GermanMexican energy partnership (EP) and florence school of regulation (FSR).

Pouttu, A. et al. (2017). "P2P model for distributed energy trading, grid control and ICT for local smart grids." In EuCNC 2017-European conference on networks and communications. https:// doi.org/10.1109/EuCNC.2017.7980652

Prettico, G., Flammini, M. G., Andreadou, N., Vitiello, S., Fulli, G., \& Masera, M. (2019). Distribution system operators observatory 2018-Overview of the electricity distribution system in Europe, EUR 29615 EN. Publications Office of the European Union.

REScoop.eu. (2019). “Q \& A : What are 'citizen' and 'renewable' energy communities ?.” https:// www.rescoop.eu/blog/what-are-citizen-and-renewable-energy-communities. Accessed July 04 2019.

REScoop.eu. (2020). https://www.rescoop.eu/. Accessed December 15, 2020.

Rivest, R. L., Adleman, L., \& Dertouzos, M. L. (1978). On data banks and privacy homomorphisms. Foundation Security Computer, 4(11), 169-180

Roberts, J., \& Gauthier, C. (2019). "Energy communities in the draft national energy and climate plans: Encouraging but room for improvements executive summary." REScoop.eu, European University Viadrina, p. 55. [Online]. Available: https://www.rescoop.eu/blog/necps

Ruelens, F., Claessens, B. J., Quaiyum, S., De Schutter, B., Babuška, R., \& Belmans, R. (2018). Reinforcement learning applied to an electric water heater: From theory to practice. IEEE Transactions Smart Grid, 9(4), 3792-3800. https://doi.org/10.1109/TSG.2016.2640184

Shoreh, M. H., Siano, P., Shafie-khah, M., Loia, V., \& Catalão, J. P. S. (2016). A survey of industrial applications of demand response. Electrical Power System Research, 141, 31-49. https://doi.org/ 10.1016/J.EPSR.2016.07.008

Siano, P. (2014). Demand response and smart grids-A survey. Renewable and Sustainable Energy Reviews, 30, 461-478. https://doi.org/10.1016/J.RSER.2013.10.022

Smart Grid Coordination Group CEN-CENELEC-ETSI. (2012). "Smart grid reference architecture". [Online]. Available: ftp://ftp.cencenelec.eu/EN/EuropeanStandardization/HotTopics/Sma rtGrids/Reference_Architecture_final.pdf

SNIPPET-Secure and privacy-friendly peer-to-peer electricity trading. (2019). https://www.esat. kuleuven.be/cosic/project/snippet/. Accessed July 10, 2019.

Synergrid. (2017). "C1/107 Algemene technische voorschriften voor de aansluiting van een gebruiker op het LS-distributienet".

Vandael, S., Claessens, B., Hommelberg, M., Holvoet, T., \& Deconinck, G. (2013). A scalable three-step approach for demand side management of plug-in hybrid vehicles. IEEE Transactions on Smart Grid, 4(2), 720-728. https://doi.org/10.1109/TSG.2012.2213847

Vandael, S., Claessens, B., Ernst, D., Holvoet, T., \& Deconinck, G. (2015). Reinforcement learning of heuristic EV fleet charging in a day-ahead electricity market. IEEE Transactions Smart Grid, 6(4), 1795-1805. https://doi.org/10.1109/TSG.2015.2393059 
Vanthournout, K., De Brabandere, K., Haesen, E., Van den Keybus, J., Deconinck, G., \& Belmans, R. (2005). Agora: Distributed tertiary control of distributed resources. 15th Power System Computer Conference, 2005, 7

Vázquez-Canteli, J. R., \& Nagy, Z. (2019). Reinforcement learning for demand response: A review of algorithms and modeling techniques. Applied Energy, 235, 1072-1089. https://doi.org/10.1016/ J.APENERGY.2018.11.002

Zhang, C., Wu, J., Long, C., \& Cheng, M. (2017). Review of existing peer-to-peer energy trading projects. Energy Procedia, 105, 2563-2568. https://doi.org/10.1016/J.EGYPRO.2017.03.737

Zhang, C., Wu, J., Zhou, Y., Cheng, M., \& Long, C. (2018). Peer-to-peer energy trading in a microgrid. Applied Energy, 220, 1-12. https://doi.org/10.1016/J.APENERGY.2018.03.010

Open Access This chapter is licensed under the terms of the Creative Commons Attribution 4.0 International License (http://creativecommons.org/licenses/by/4.0/), which permits use, sharing, adaptation, distribution and reproduction in any medium or format, as long as you give appropriate credit to the original author(s) and the source, provide a link to the Creative Commons license and indicate if changes were made.

The images or other third party material in this chapter are included in the chapter's Creative Commons license, unless indicated otherwise in a credit line to the material. If material is not included in the chapter's Creative Commons license and your intended use is not permitted by statutory regulation or exceeds the permitted use, you will need to obtain permission directly from the copyright holder.

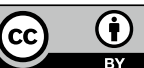

